\title{
Predicting swimming performance using state anxiety
}

\author{
Smart Z. Mabweazara, Lloyd Leach and Barry S. Andrews
}

\begin{abstract}
Competitive state anxiety is a common response to stressful competitive sports situations that could affect athletic performance. The effects of state anxiety on swimming performance need further inquiry. The aim of the study was to determine the component of state anxiety that best predicts swimming performance. A quantitative, cross-sectional study design that made use of the Competitive State Anxiety Inventory-2 to measure precompetitive state anxiety was used. A total of 61 male high school swimmers whose age ranged between 14 and 19 years $(M=16.16$, standard deviation $=1.66$ years $)$ completed the Competitive State Anxiety Inventory-2 $1 \mathrm{hr}$ before competing in a 50-m individual swimming event. Performance was evaluated using finishing position. Due to the relatively short duration of the $50-\mathrm{m}$ event, the available literature would suggest that Somatic Anxiety would have a greater effect on Performance-there is not enough time to allow cognitive anxiety to have a detrimental impact on performance. Thus, it was hypothesized that somatic rather than cognitive anxiety will best predict swimming performance. It emerged that both cognitive $(b=.787 ; p<.001)$ and somatic anxieties $(b$ $=.840 ; p<.001$ ) can independently predict swimming performance. However, when both cognitive and somatic anxieties were regressed onto swimming performance, somatic anxiety partially dominated cognitive anxiety $(b=.626 ; p<.001)$ and became the significant predictor of swimming performance. It is recommended that swimmers and swimming coaches make use of specific intervention strategies that eradicate the detrimental effects of somatic anxiety immediately before competition.
\end{abstract}

Anxiety and arousal are two related psychological constructs that have been shown to affect athletic performance. Arousal refers to a blend of physiological processes in a person that are related to the intensity dimensions of motivations at a particular moment (Weinberg \& Gould, 2011). On the other hand, precompetitive state anxiety may be regarded as anxiety prior to the onset of competition and is reflective of the objective and perceived requirements of the individual or team participating in the competition (Raglin \& Morris, 1994). Novice athletes seem to experience a steady increase in anxiety in the immediate period leading up to and during competition, while experienced athletes may have increased anxiety during precompetition periods further away from competition and a decrease in the immediate period leading up to and during competition (Bridges \& Knight, 2005). Precompetitive anxiety is usually caused by the psychological pressure induced by both the presence and expectations of significant others such as coaches and parents. Weinberg and Gould (2011) have stated that the incapability to cope with the pressure in competitive sport can lead to poor performance and at times even 
deteriorate to physical illness and mental distress. Martens, Vealey, and Burton (1990b) provided a multidimensional approach to the inquiry of anxiety. Their multidimensional theory proposes that anxiety is sub-composed of cognitive and somatic anxiety. They defined cognitive anxiety as "the mental component of anxiety" (Martens et al., 199ob, p. 6). Cognitive anxiety was said to have a negative linear relationship with performance. Somatic anxiety was defined as "the physiological and affective elements of the anxiety experience that develop directly from autonomic arousal" (Martens et al., 199ob, p. 6). The authors proposed that somatic anxiety affects performance in a curvilinear fashion, with both lower and higher levels of somatic anxiety being debilitative to performance. It was also posited that somatic anxiety reaches its maximum levels at the beginning of competition and begins to fade once the contest starts. As such, somatic anxiety was said to have a minimal impact on performance compared to cognitive anxiety (Martens, Burton, Vealey, Bump, \& Smith, 1990a). However, this depends on the duration of the event. Specifically for this study, due to the short time taken to complete the $50-\mathrm{m}$ swim, the impact of somatic anxiety might be greater than that of cognitive anxiety. For example, Burton (1988) found that short duration events demonstrate stronger relationships between somatic anxiety and performance than longer events. To further differentiate the two constructs, cognitive anxiety, which affects the mental element, is expressed during self-evaluation and self-talk, while somatic anxiety, which is the physiological element, is expressed as autonomic arousal (Parnabas, Mahamood, \& Parnabas, 2013).

The relationship between cognitive and somatic state anxiety as predictors of performance in different sports is a function of the specific motoric and physiological requirements of the athletic activity (Taylor, 2006). Thus, sports that involve strength, such as weightlifting, require elevated levels of state anxiety and physiological arousal, whereas sports that are more skill-oriented, such as archery, require low arousal and anxiety levels for peak performance (Taylor, 2006). As such, swimming strokes that are more strenuous will require elevated arousal and anxiety levels, whereas strokes that are less demanding in terms of strength will require less arousal and anxiety levels. In other activities, such as performance in music, the results have been somewhat different. Yoshie, Shigemasu, Kudo, and Ohtsuki (2009) investigated the effects of state anxiety on music performance. The results showed that the magnitude of cognitive anxiety negatively predicted technical accuracy, while cognitive anxiety positively predicted global performance. The authors concluded that reducing pre-performance cognitive anxiety and understanding the symptoms of cognitive anxiety as being facilitative to performance could enhance performance. This finding, although it relates to music performance, may also apply to swimming performance; in other words, swimmers must be able to minimize pre-performance cognitive anxiety in order to enhance their swimming performance. Taylor (2006) further notes that extreme levels of state anxiety impede motor coordination and the effective use of the cardiovascular system.

Currently, the literature is equivocal about the effect of anxiety on performance (Polman, Rowcliffe, Borkoles, \& Levy, 2007). Some studies (Burton, 1988; Jones et al., 1994) have concluded that state anxiety has a debilitating effect on swimming performance, whereas other studies (Furst \& Tenebaum, 1986) have found state anxiety to be facilitative of swimming performance. Polman et al. (2007) concluded that state 
anxiety is both debilitative and facilitative to swimming performance. Nordell and Sime (1993) have also reported that other studies have found no association between swimming performance and state anxiety. Lundqvist, Kentta, and Raglin (2011) examined the differences in anxiety ratings of elite swimmers on the direction modified Competitive State Anxiety Inventory-2R. They showed that elite athletes rated more items on the scale as facilitative to performance, whereas sub-elite athletes rated more items on the scale as debilitative.

Females are reported to display significantly elevated levels of predicted precompetition anxiety than males (Wilson, Raglin, \& Pritchard, 2002). However, the authors point out that gender is a poor predictor of precompetition anxiety. Although this is the case, the significance of Wilson et al.'s (2002) findings is that state anxiety research about one gender should not overlap into that of the other gender. As such, research about the state anxiety should focus on one gender as is the case in this study. Wilson et al. (2002) also reported that optimistic college athletes exhibit significantly lower levels of precompetition anxiety compared to pessimists. A sound explanation of this finding could be that optimistic athletes are more self-confident compared to pessimistic athletes. As a result, numerous sport psychology interventions for performance enhancement typically include techniques designed to enhance self-confidence (Weinberg \& Gould, 2011) and reduce anxiety (LeUness \& Nation, 2002). The predictability of swimming performance from state anxiety measures remains unclear. Furthermore, it is not clear which type of anxiety best predicts swimming performance.

Much more recently, using the same participants and research instruments as in this study, Mabweazara, Andrews, and Leach (2014) assessed the temporal changes in state anxiety among high school swimmers prior to competition. The Competitive State Anxiety Inventory-2 (CSAI-2) was used to measure state anxiety 7 days (baseline measures) before a major competition and $1 \mathrm{hr}$ (precompetition measures) before the competition, under the supervision of a registered psychologist. Significant differences between baseline and precompetitive cognitive state anxieties $(p=.000)$ and baseline and precompetitive somatic anxieties $(p=.000)$ were reported. The authors concluded that state anxiety significantly increases prior to competition among high school swimmers. The study clearly showed the importance of assessing state anxiety among swimmers, especially before a major competition by making use of a real competitive situation as compared to creating a pseudo-competitive environment, which might not be perceived as competitive by the young swimmer. It is in line with this, that this study assesses the predictability of performance using state anxiety among swimmers within the same competitive context. Coaches and swimmers should thus make use of specific psychological intervention strategies in order to be able to regulate state anxiety and change potentially debilitative anxiety to facilitative state anxiety. Given the foregoing, this study extends and builds on the work of Mabweazara et al.'s (2014) study and shifts focus from assessing the temporal changes in state anxiety prior to competition in determining the component of state anxiety that best predicts swimming performance using precompetitive state anxiety measures. It was hypothesized that somatic rather than cognitive state anxiety significantly predicts swimming performance. 


\section{Method}

The study utilized a quantitative, descriptive design.

\section{Participants}

Male high school swimmers comprised the study population. Purposive sampling was used to identify 61 male swimmers from three private High Schools in Zimbabwe. The following inclusion criteria were used: swimmers registered as High School learners at selected private schools in Zimbabwe; swimmers whose ages ranged from 15 to 19 years; swimmers who competed in the Falcon College Schools Swimming Gala (16 October 2012); swimmers who competed in the individual $50 \mathrm{~m}$ front crawl, back stroke, breast stroke, or butterfly swimming events; swimmers who competed at provincial level; swimmers with at least 4 years' experience in competitive swimming. One school was selected from the Matabeleland South Province, while the other two schools were selected from Bulawayo Province. Private schools were specifically chosen for this study because in Zimbabwe, specifically in the Province where the study was undertaken, most public schools cannot afford the luxury of a swimming pool because installing and maintaining one is expensive. As such, it is mostly private schools that can afford having a swimming pool and hiring coaching staff. The three High Schools included in this study were chosen on the basis that they are the most competitive.

\section{Instruments}

Precompetitive state anxiety was measured using the CSAI-2, a questionnaire developed by Martens et al. (1990b). The CSAI-2 is a 27-item self-report measure of anxiety with three 9-item subscales that measure cognitive state anxiety, somatic state anxiety, and state self-confidence (Duda, 1998). In this study, the state self-confidence subscale of the CSAI-2 was excluded. The rationale for using the CSAI-2 was that the instrument has been used in a landmark study by Burton (1988), which utilized elite swimmers to show the reliability of the CSAI-2 in terms of stability and consistency. The CSAI-2 was administered $1 \mathrm{hr}$ prior to competition. Burton's (1998) landmark study also provided confirmation for the theoretical underpinnings of the multidimensional theory. As in Burton's (1988) study, this study utilized the CSAI-2 to measure state anxiety just prior to competition. Initial psychometric studies on the CSAI-2 inventory have demonstrated an internal consistency for each of the three subscales (Martens et al., 199ob). Separate alpha coefficients for the cognitive, somatic, and self-confidence subscales ranged from .79 to .90 thereby revealing a high degree of internal consistency (Martens et al., 1990b). Other studies employing the CSAI-2 to measure anxiety in sport have reported alpha reliability coefficients that range from .76 to .91 , thereby confirming its internal consistency (Duda, 1998, p. 136). Similarly, in an African setting, Adeniyi and Samson (2004) have established the reliability of this multidimensional measure of state anxiety with Nigerian athletes and have documented an $r$ of .80. The reliability coefficients for the scales in this study were satisfactory (Cronbach alpha scores of .68 and .80) for the cognitive and somatic anxiety scales, respectively.

In this research, any scores that were less than or equal to 12 points were considered to be low for state anxiety, whereas scores ranging from 13 to 24 points were considered to be medium and scores ranging from 25 to 36 points were considered to be high anxiety scores. This was done for convenience and in line with Martens et al.'s (1990a) 
instructions that on the CSAI-2 inventory, state anxiety scores range from a minimum of 9 to a maximum of 36 .

\section{Procedure}

The swimmers were assessed at an Inter-Schools Swimming Gala (2012) in Zimbabwe. With the assistance of the swimming coaches from each of the participating schools, a premier inter-schools swimming competition was identified. This swimming competition was assumed to bring out the competitive character of the swimmers. Such an environment would likely lead to the swimmers experiencing precompetitive state anxiety (Gill, 1986). A brief explanation of the study was then provided by the investigator to the school authorities, coaches, and participants, and participants' parents. It was important in this study to collect anxiety measures twice in order to observe whether there was a change in the state anxiety of swimmers from a non-competitive context to a competitive context. This was done so that the precompetitive state anxiety levels observed an hour before the competition could be attributed to the competitive nature of the environment. Therefore, the precompetitive state anxiety of the swimmers was first assessed 7 days prior to the competition in a non-competitive context during training at their respective schools using the CSAI-2. This assessment was used as a baseline for comparison purposes. Each swimmer then completed the CSAI-2 inventory on the day of competition, an hour before competing in an individual $50 \mathrm{~m}$ swimming event, that is, the front crawl, butterfly, breast, and/or back stroke. On each occasion, before the swimmers completed the questionnaire, the CSAI-2 instructions were read aloud to them. The final results of the swimmers' performances were e-mailed by the head coach of the host school to the investigator 3 days after the competition.

Race finishing position was used to evaluate performance. The finishing positions in the swimming competitions were categorized by convenience in order to facilitate data comparisons. Therefore, Positions 1 and 2 were categorized as being above average performance, with Positions 3 and 4 as average performance and Positions 5 and 6 as below average performance. The swimmers were then categorized as being of above average, average, or below average performance. This was done for convenience.

\section{Ethical considerations}

Informed consent for participation in the study was obtained from the participants. Consent was also obtained from the parents of the participants. Participation in the study was voluntary and the participants could withdraw from the study at any point without coercion or any negative consequences. Permission to conduct the study and ethical approval for the study was obtained from the Ethics Committee of the University of the Western Cape, the Zimbabwean Ministry of Education, Sport, Arts and Culture, and the three private schools selected in the Bulawayo and Matabeleland South Provinces (ethics clearance number 12-7-9).

\section{Data analysis}

Descriptive and inferential statistics were used on SPSS (version 21) to analyze the data. Means and standard deviations were calculated for precompetitive state anxiety intensity levels, swimming performance, and the ages of the participants. Spearman correlations were computed to test for significant associations between precompetitive 
state anxiety and swimming performance. Hierarchical regression analyses were performed to show the strength of association of each of the subcomponents of state anxiety with swimming performance and to show the component of precompetitive state anxiety that best predicts swimming performance. Statistical significance was set at $p$ $<$.05. Data are presented using tables.

\section{Results}

The participants' $(n=61$ ) ages ranged from 14 to 19 years (mean $=16.16$, standard deviation $[S D]=1.66$ years). Performance placement fell into six categories, that is, from positions 1 to 6 . In all, 10 participants (16.39\%) finished in first position, 9 (14.75\%) in the second position, 12 (19.67\%) in the third position, 10 (16.39\%) in the fourth position, $11(18.03 \%)$ in the fifth position, and $9(14.75 \%)$ in the sixth position. Table 1 shows the performance placement in terms of finishing position of the participants in frequencies and percentages.

Table 1. Performance placement of the participants expressed as frequencies and percentages

\begin{tabular}{lcc}
\hline Placement & Frequency & Percentage \\
\hline 1 & 10 & 16.39 \\
2 & 9 & 14.75 \\
3 & 12 & 19.67 \\
4 & 10 & 16.39 \\
5 & 11 & 18.03 \\
6 & 9 & 14.75 \\
Total & 61 & 100 \\
\hline
\end{tabular}

Table 2. Precompetitive cognitive and somatic state anxiety of swimmers based on finishing position.

\begin{tabular}{lccc}
\hline $\begin{array}{l}\text { Ranking } \\
\text { Finishing position }\end{array}$ & $N$ & $\begin{array}{l}\text { Cognitive anxiety } \\
(\text { mean } \pm \text { SD) }\end{array}$ & $\begin{array}{l}\text { Somatic anxiety } \\
(\text { mean } \pm \text { SD) }\end{array}$ \\
\hline $\begin{array}{l}\text { Above average } \\
\text { (Positions I and 2) }\end{array}$ & 19 & $13.05 \pm 2.39$ & $18.05 \pm 1.99$ \\
$\begin{array}{l}\text { Average } \\
\text { (Positions 3 and 4) }\end{array}$ & 22 & $24.77 \pm 2.31$ & $24.32 \pm 2.00$ \\
$\begin{array}{l}\text { Below average } \\
\text { (Positions 5 and 6) }\end{array}$ & 20 & $27.10 \pm 4.05$ & $28.15 \pm 2.85$ \\
\hline
\end{tabular}

SD: standard deviation.

Table 3. State anxiety scores at precompetition.

\begin{tabular}{|c|c|c|c|c|c|c|c|c|}
\hline \multirow[t]{2}{*}{ Period } & \multicolumn{2}{|c|}{ High } & \multicolumn{2}{|c|}{ Medium } & \multicolumn{2}{|c|}{ Low } & \multicolumn{2}{|c|}{ Total } \\
\hline & $n$ & $\%$ & $n$ & $\%$ & $n$ & $\%$ & $n$ & $\%$ \\
\hline Precompetitive cognitive anxiety & 27 & 44.26 & 26 & 42.62 & 8 & 13.11 & 61 & 100 \\
\hline Precompetitive somatic anxiety & 31 & 50.82 & 30 & 49.18 & 0 & 0.00 & 61 & 100 \\
\hline
\end{tabular}

Table 2 shows the precompetitive cognitive and somatic state anxiety mean scores for the three performance groups, namely, swimmers with above average (Positions 1 and 2), average (Positions 3 and 4), and below average (Positions 5 and 6) finishing performance. The mean $\pm S D$ scores for the above average group for cognitive and somatic anxiety 
were $13.05 \pm 2.39$ and $18.05 \pm 1.99$, respectively. The mean $\pm S D$ scores for the average group for cognitive and somatic anxiety were $24.77 \pm 2.31$ and $24.32 \pm 2.00$, respectively. The mean $\pm S D$ scores for the below average group for cognitive and somatic anxiety were $27.10 \pm 4.05$ and $28.15 \pm 2.85$, respectively.

Table 3 shows the state anxiety scores at precompetition (i.e., $1 \mathrm{hr}$ before competition) categorized into high, medium, and low frequencies and percentages. In total, 27 participants (44.26\%) experienced high cognitive state anxiety, 26 (42.62\%) experienced medium, and 8 (13.11\%) participants experienced low cognitive state anxiety. In all, 31 participants (50.82\%) experienced high somatic state anxiety, 30 (49.18\%) experienced medium, and none of the participants scored low on the somatic state anxiety scale $1 \mathrm{hr}$ before competition.

Table 4 shows the correlation matrix for performance (finishing placement) and precompetitive state anxiety. Specifically, there was a significant correlation $(p<.001)$ between precompetitive cognitive state anxiety and performance $(r=.787)$. The results also indicate a significant correlation $(p<.001)$ between precompetitive somatic state anxiety and swimming performance $(r=.873)$.

Table 4. Correlation matrix for placement and precompetitive state anxiety.

\begin{tabular}{|c|c|c|c|c|}
\hline \multirow[t]{2}{*}{ Variables } & \multicolumn{4}{|c|}{ Variables } \\
\hline & I & 2 & 3 & 4 \\
\hline I. Age & - & & & \\
\hline 2. Cognitive anxiety & .181 & - & & \\
\hline 3. Somatic anxiety & .058 & .825 *** & - & \\
\hline 4. Placement & .028 & $.787^{* * * *}$ & $.873 * *$ & - \\
\hline
\end{tabular}

Table 5. Model summary of the hierarchical regression in five steps.

\begin{tabular}{llllllll}
\hline Step & Criterion & Outcome & $b$ & Partial & Excluded & $R^{2}$ & \multicolumn{1}{l}{} \\
\hline $\mathrm{I}$ & Cognitive anxiety & Somatic anxiety & $.850^{* * *}$ & & & .721 & $152.768^{* * *}$ \\
2 & Somatic anxiety & Cognitive anxiety & $.850^{* * *}$ & & & .721 & $152.768^{* * *}$ \\
3 & Somatic anxiety & Placement & $.840^{* *}$ & & & .710 & $144.434^{* * *}$ \\
4 & Cognitive anxiety & Placement & $.787^{* * *}$ & & & .619 & $95.896^{* * *}$ \\
5 & Cognitive anxiety & Placement & .255 & .250 & & .728 & $77.679 * *$ \\
& Somatic anxiety & Placement & $.626^{* *}$ & $.535^{* *}$ & Cognitive anxiety & & \\
\hline
\end{tabular}

b (standardized regression coefficients) is a measure of how strongly each predictor (outcome) variable influences the criterion variable.

$* * p<.001$.

The results also indicate a significant correlation $(p<.001)$ between precompetitive cognitive state anxiety and somatic state anxiety $(r=.825)$. Performance or finishing position was coded so that faster finishing order denoted lower rankings. Both correlations were large and positive, that is, as anxiety increased, performance or finishing position increased. In other words, the positive correlation coefficients actually indicates an inverse relationship between state anxiety and performance. There was a 
significant negative relationship between precompetitive state anxiety and performance, that is, as anxiety increased, performance decreased.

Hierarchical regression analysis was performed in order to establish the component of state anxiety that best predicts swimming performance. The hierarchical regression analysis results are shown in Table 5 .

When somatic state anxiety was regressed onto cognitive state anxiety and vice versa, identical results were found. Both models were significant $(p<.001)$ and explained $72 \%$ $\left(R^{2}\right)$ of the variance in cognitive state anxiety.

When somatic anxiety was regressed onto placement, the result was significant $(p<.001)$ and explained $71 \%\left(R^{2}\right)$ of the variance in finishing position. Somatic state anxiety was a significant predictor of placement $(p<.001)$, that is, for every 1-unit change in somatic state anxiety, there was a corresponding change of 0.84 units in finishing position.

When cognitive state anxiety was regressed onto placement, the result was significant $(p<$ .oo1) and explained $62 \%\left(R^{2}\right)$ of the variance on finishing position. Cognitive state anxiety was a significant predictor of placement $(p<.001)$, that is, for every 1-unit change in cognitive state anxiety, there was a corresponding change of 0.78 units in finishing position.

When both cognitive state anxiety and somatic state anxiety were regressed onto placement, the result was significant $(p<.001)$ and explained $73 \%\left(R^{2}\right)$ of the variance on finishing position. In this final step, cognitive state anxiety did not significantly predict performance. Consequently, somatic state anxiety became the dominant predictor of performance.

Three important observations emerge from this analysis (see Table 5):

1. Somatic state anxiety and cognitive state anxiety are in a linear relationship and can predict each other significantly (Steps 1 and 2).

2. Somatic state anxiety and cognitive state anxiety both independently predict placement significantly (Steps 3 and 4).

3. Somatic state anxiety dominates cognitive state anxiety in the mixed model (Step 5).

Thus, in the final step of the analysis, cognitive state anxiety was excluded from the hierarchical regression in which case somatic state anxiety became the significant predictor of performance. It therefore emerged that somatic state anxiety is a manifestation of cognitive state anxiety or acts in place for cognitive state anxiety (proxy relationship).

\section{Discussion}

In this study, it was hypothesized that cognitive state anxiety rather than somatic state anxiety would be the best predictor of swimming performance. When somatic state 
anxiety was regressed onto performance, it showed to be a significant predictor of performance. Cognitive anxiety was also a significant predictor of performance; however, in the last step of the hierarchical regression, when both cognitive and somatic state anxieties were regressed onto performance, somatic state anxiety became the only significant predictor of swimming performance. It was thus concluded that somatic state anxiety acts in place of cognitive anxiety (a proxy relationship) or is manifestation of cognitive state anxiety. This is reflected in the results showing that both cognitive and somatic state anxieties significantly predict each other.

Studies conducted by Edwards and Hardy (1996) and Jerome and Williams (2000) have also found that competitive anxiety intensity is a significant predictor of performance variance. However, Martens et al. (1990b) hypothesized that cognitive state anxiety would be a stronger predictor of performance than somatic state anxiety. However, it is not clear whether these conclusions about somatic anxiety and cognitive anxiety relationship with performance, were made with the duration of events taken into consideration. The findings of this study are contradictory to this assertion. Several other research findings, which contradict the present findings, are in agreement with the prediction that cognitive anxiety is a stronger predictor of performance than somatic anxiety (Lane, Terry, \& Karageorghis, 1995a, 1995b). It is, however, worth noting that the study by Lane et al. (1995b) employed male triathlete participants, and as such, their findings might have been influenced by the long duration of the triathlon event.

In a study utilizing intra-individual measures, which was rooted in multidimensional theory, Burton (1988) predicted performance to decline linearly with surges in cognitive state anxiety. In general, research concurs with the assertion that cognitive anxiety is linked to factors in the environment which impact perceptions of success or failure (Gould, Petlichkoff, \& Weinberg, 1984; Hanton \& Jones, 1995; Jones, Swain, \& Cale, 1990; Lane et al., 1995a, 1995b). A number of explanations have been given for these contradictions, including "the use of poor performance measures, failure to control for individual differences in ability, response sensitivity, anxiety tolerance, and failure to consider subjects' interpretations of the meaning of their anxiety symptoms" (Hardy, Jones, \& Gould, 1996, p. 149).

Craft, Magyar, Becker, and Feltz (2003) specifically observe that the use of the CSAI-2 has not managed to yield stable results in terms of predicting performance. This study used an objective outcome (finishing position) as a performance measure. However, it has been noted that the most dominant methodological limitations in examining the anxiety-performance relationship have been complications with the working definitions of performance (Krane, 1992). Performance may be evaluated in terms of objective results, such as finishing position, or subjective outcomes, such as evaluation by a coach, or even based on self-perceptions of performance (Craft et al., 2003). These different methods of evaluating performance may yield different results (Craft et al., 2003).

This study attempted to determine the strongest predictor of swimming performance in the 50-m events. Martens et al. (1990a) probed the impact of task characteristics on the relationship between cognitive and somatic anxiety and swimming performance. For 
the 50-m freestyle event, the results of Martens et al.'s (1990a) study were similar to those of this study. Somatic state anxiety was significantly related to performance. Martens et al. (1990a) recommend that somatic state anxiety is a central mediator of sprint freestyle performance. As in this study, somatic state anxiety played a significant role in predicting performance for short or sprint events in swimming.

In this study, the results suggested that somatic anxiety is merely a manifestation of cognitive state anxiety or that it acts as a proxy for cognitive state anxiety. Although these two components of anxiety are theoretically autonomous, Morris, Davies, and Hutchings (1981) have recommended that they co-vary in demanding situations such as competition since these situations contain elements related to the manifestation of both somatic and cognitive reactions. A weak reliance of cognitive anxiety on somatic anxiety has been shown in a number of studies (Deffenbacher, 1980; Morris \& Liebert, 1973). Borkovec (1976) suggests that each constituent of anxiety may serve a provisional or discriminative function for the other constituent.

If strong somatic reactions (e.g., heavy sweating) by an athlete are linked to a certain provocation or stimulus present in the competition arena (e.g., a highly competitive sport setting), these reactions may indicate to a person that there is reason to worry (Martens et al., 1990a). A swimmer, for example, may acquire conditioned somatic anxiety reactions, because of precompetitive activities, such as the precompetition team talk or the mere presence of noisy spectators. These somatic anxiety reactions may then prompt the swimmer to start worrying. In other words, the somatic anxiety reactions incite cognitive anxiety. This may also happen the other way round where cognitions, manifested through undesirable pictures of failure, may generate a specific form of somatic response. In this study, somatic state anxiety became a proxy for cognitive state anxiety, probably due to the presence of a highly evaluative crowd that is made up of coaches, provincial team scouts, parents, spectators, and teachers.

Knowing that anxiety will have an effect on performance is widely accepted in sport, but knowing that somatic anxiety (the physiological arousal levels) has the greatest impact on performance can affect the coaching of swimmers. Coaches can plan and instruct their swimmers on ways to cope with somatic anxiety, and also to be on the lookout for it so as to negate its effects on the athlete's performance, thereby assisting their swimmers to be more successful.

Transferability of the findings of this study is limited because of the non-randomized recruitment of subjects. Also, this study did not assess whether the swimmers interpreted the experienced state anxiety as either facilitative or debilitative to swimming; rather it focused on the influence of anxiety intensity on swimming performance. It has been recommended that researchers consider examining the direction dimension of state anxiety on sport performance (Kais \& Raudsepp, 2005). This study quantified performance as a function of finishing positions. Researchers are encouraged to use world age group swimming record for specific events. 


\section{Conclusion}

This study reveals that both cognitive and somatic anxiety can independently predict swimming performance. However, somatic state anxiety was the stronger predictor of performance. We thus conclude that somatic anxiety has the greatest impact on sprint performance (shorter swimming events). It is thus recommended that coaches and swimmers use specific intervention strategies that eradicate the detrimental effects of somatic anxiety on swimming performance in order to optimize performance.

\section{Funding}

The author(s) received no financial support for the research, authorship, and/or publication of this article. 


\section{References}

Adeniyi, A. M., \& Samson, A. B. (2004). Predictors of pre-game anxiety dysphoria among teenage soccer players. Nigerian Journal of Emotional Psychology, 6, 1518.

Borkovec, T. D. (1976). Physiological and cognitive process in the regulation of anxiety. In G. E. Schwartz \&

D. Shapiro (Eds.), Consciousness and self-regulation (Advances in Research and Theory, pp. 216-312). New York, NY: Plenum.

Bridges, A., \& Knight, B. (2005). The role of cognitive and somatic anxiety in athletic performance. $\quad$ Retrieved

from http://psych.hanover.edu/research/Thesiso5/BridgesKnight.pdf

Burton, D. (1988). Do anxious swimmers swim slower? Re-examining the elusive anxiety performance rela- tionship. Journal of Sport and Exercise Psychology, 10, 45-61.

Craft, L. L., Magyar, T. M., Becker, B. J., \& Feltz, D. (2003). The relationship between the Competitive State Anxiety Inventory-2 and sport performance: A meta-analysis. Journal of Sport and Exercise Psychology, 25, 44-65.

Deffenbacher, J. L. (1980). Worry and emotionality in test anxiety. In I. G. Sarason (Ed.), Test anxiety: Theory, research and applications (pp. 111-124). Hillsdale, NJ: Lawrence Erlbaum.

Duda, J. (1998). Advances in sport and exercise psychology measurement. Morgantown, WV: Fitness Information Technology.

Edwards, T., \& Hardy, L. (1996). The interactive effects of intensity and direction of cognitive and somatic anxiety and self-confidence upon performance. Journal of Sport and Exercise Psychology, 18, 296-312.

Furst, D. M., \& Tenebaum, G. (1986). The relationship between worry, emotionality and sport performance. In D. M. Landers (Ed.), Sport and elite performers (pp. 8996). Champaign, IL: Human Kinetics.

Gill, D. L. (1986). Psychological dynamics of sport. Champaign IL: Human Kinetics.

Gould, D., Petlichkoff, L., \& Weinberg, R. S. (1984). Antecedents of temporal changes in and relationships between CSAI-2 subcomponents. Journal of Sport and Exercise Psychology, 6, 289-304.

Hanton, S., \& Jones, G. (1995). Antecedents of anxiety and confidence in elite competitive swimmers. International Journal of Sport Psychology, 26, 512-523.

Hardy, L., Jones, J. G., \& Gould, D. (1996). Understanding psychological preparation for sport. Chichester, UK: John Wiley \& Sons.

Jerome, G. J., \& Williams, J. M. (2000). Intensity and interpretation of competitive state anxiety: Relationships to performance and repressive coping. Journal of Applied Sport Psychology, 12, 236-250.

Jones, G., Swain, A., \& Cale, A. (1990). Antecedents of multidimensional competitive state anxiety and self-confidence in elite intercollegiate middle-distance runners. The Sport Psychologist, 4, 107-118.

Jones, G., Hanton, S., \& Swain, A.B.J. (1994). Intensity and interpretation of anxiety symptoms in elite and non-elite sports performers. Personal and individual Differences, 17, 657-663.

Kais, K., \& Raudsepp, L. (2005). Intensity and direction of competitive state anxiety, self-confidence, and athletic performance. Kinesiology, 37, 13-20. 
Krane, V. (1992). Conceptual and methodological considerations in sport anxiety research: From the inverted-U hypothesis to catastrophe theory. Quest, 44, 72-87.

Lane, A. M., Terry, P. C., \& Karageorghis, C. I. (1995a). The antecedents of multidimensional state anxiety and self-confidence in duathletes. Perceptual and Motor Skills, 8o, 911-919.

Lane, A. M., Terry, P. C., \& Karageorghis, C. I. (1995b). Path analysis examining relationships among antecedents of anxiety, multidimensional state anxiety, and triathlon performance. Perceptual and Motor Skills, 81, 1255-1266.

LeUness, A., \& Nation, J. R. (2002). Sport psychology. Louisville, KY: Wordsworth Group.

Lundqvist, C., Kentta, G., \& Raglin, J. S. (2011). Directional anxiety responses in elite and sub-elite young athletes: Intensity of anxiety symptoms matters. Scandinavian Journal of Medicine and Science in Sports, 21, 853-862.

Mabweazara, S. Z., Andrews, B. S., \& Leach, L. L. (2014). Changes in state anxiety prior to competition. African Journal for Physical, Health Education, Recreation and Dance, 2O, 492-499.

Martens, R., Burton, D., Vealey, R. S., Bump, L. A., \& Smith, D. E. (1990a). Development and validation of the Competitive State Anxiety Inventory-2 (CSAI-2). In R. Martens, R. S. Vealey, \& D. Burton (Eds.), Competitive anxiety in sport (pp. 193208). Champaign, IL: Human Kinetics.

Martens, R., Vealey, R. S., \& Burton, D. (1990b). Competitive anxiety in sport. Champaign, IL: Human Kinetics.

Morris, L. W., Davies, D., \& Hutchings, C. (1981). Cognitive and emotional components of anxiety: Literature review and revised worry-emotionality scale. Journal of Educational Psychology, 73, 541-555.

Morris, L. W., \& Liebert, R. M. (1973). Effects of negative feedback, threat of shock, and level of trait anxiety on the arousal of two components of anxiety. Journal of Counseling Psychology, 2O, 321-326.

Nordell, K. A., \& Sime, W. (1993). Competitive trait anxiety, state anxiety, and perceptions of anxiety, state anxiety, and perceptions of anxiety: Interrelationships in practice and in competition. The Journal of Swimming Research, 9, 19-24.

Parnabas, V. A., Mahamood, Y., \& Parnabas, J. (2013). The relationship between cognitive and somatic anxiety on performance of student-athletes of Universiti Malaysia Perlis (UNIMAP). Sport and Art, 1(3), 61-66.

Polman, R., Rowcliffe, N., Borkoles, E., \& Levy, A. (2007). Precompetitive state anxiety, objective and subjective performance and causal attributions in competitive swimmers. Pediatric Exercise Science, 19, 39-50.

Raglin, J. S., \& Morris, M. J. (1994). Precompetition anxiety in women volleyball players: A test of ZOF theory in a team sport. British Journal of Sports Medicine, 28, 47-51.

Taylor, J. (2006). Predicting athletic performance with self-confidence and somatic and cognitive anxiety as a function of motor and physiological requirements in six sports. Journal of Personality, 55, 139-153.

Weinberg, R., \& Gould, D. (2011). Foundations of sport and exercise psychology. Champaign, IL: Human Kinetics. 
Wilson, G. S., Raglin, J. S., \& Pritchard, M. E. (2002). Optimism, pessimism, and precompetitive anxiety in college athletes. Personality and Individual Differences, 32, 893-902.

Yoshie, M., Shigemasu, K., Kudo, K., \& Ohtsuki, T. (2009). Effects of state anxiety on music performance: Relationship between the Revised Competitive State Anxiety Inventory-2 Subscales and Piano performance. Musicae Scientiae, 13, 55-84. 